\title{
Neuroimaging signature associated with symptom exacerbation in early-stage psychosis
}

\author{
Marina Mihaljevic ${ }^{1}$, Anisha Nagpal $^{1}$, Semra Etyemez $^{1}$, Zui Narita $^{1}$, \\ Anna Ross ${ }^{1}$, Rebecca Schaub ${ }^{1}$, Nicola Cascella ${ }^{1}$, Jennifer M. Coughlin ${ }^{1,2}$, Gerald Nestadt ${ }^{1}$, \\ Frederik C. Nucifora ${ }^{1}$, Thomas W. Sedlak ${ }^{1}$, Vince Calhoun ${ }^{7}$, Andreia Faria $^{2}$, Kun Yang ${ }^{1 *}$, \\ and Akira Sawa ${ }^{1,3,4,5,6^{*}}$
}

Departments of Psychiatry ${ }^{1}$, Radiology and Radiological Sciences ${ }^{2}$, Neuroscience ${ }^{3}$, Biomedical Engineering ${ }^{4}$, and Genetic Medicine ${ }^{5}$, Johns Hopkins University School of Medicine, Baltimore, MD. Department of Mental Health ${ }^{6}$, Johns Hopkins Bloomberg School of Public Health, Baltimore, MD. Tri-institutional Center for Translational Research in Neuroimaging and Data Science (TReNDS) ${ }^{7}$, Georgia State University, Georgia Institute of Technology, Emory University, Atlanta, GA.

*Correspondence authors

Akira Sawa (contact) : asawa1@jhmi.edu; Kun Yang: kunyang@jhmi.edu

Abstract: 207 words

Main text: 3462 words

Short/running title: Brain alterations associated with psychosis

Keywords: symptom exacerbation, relapse, early stage, first episode psychosis, resting-state functional MRI, large-scale brain network, dorsal anterior cingulate cortex, thalamus. 


\section{ABSTRACT}

Recent reports have indicated that the occurrence of symptom exacerbation in early-stage psychosis could result in brain changes, which are likely to underlie the poorer disease outcome. Thus, it is important to identify neuroimaging signature associated with symptom exacerbation in early-stage psychosis. We studied 85 patients with psychosis within two years after onset and 94 healthy controls (HC). The patient group was subdivided into two groups: 54 patients who did not experience major symptom exacerbation between the onset and study enrollment (P1), and 31 patients who experienced major symptom exacerbation (P2). We analyzed three brain imaging measures derived from resting-state functional MRI, such as global efficiency, nodal efficiency, and resting-state functional connectivity (rs-FC). After excluding some brain imaging measures that were potentially affected by clinical variables, we conducted a comparison between overall patient group and $\mathrm{HC}$ group as well as comparsions between $\mathrm{HC}, \mathrm{P} 1$, and P2 groups for these three types of brain imaging measures, respectively. By integrating the information, we pinned down the dorsal anterior cingulate cortex and thalamus as key hubs in the context of several large-scale brain networks associated with symptom exacerbations in earlystage psychosis. Our study implies the importance of considering neural mechanism associated with symptom exacerbations in early stages of psychotic disorders. 


\section{INTRODUCTION}

The trajectory of psychotic disorders after onset is heterogeneous, and many cases show deteriorating courses. ${ }^{1,2}$ One of the major determinants of poorer prognosis is occurrence of symptom exacerbations, ${ }^{3,4}$ which may be caused by intrinsic vulnerability but also by nonadherence to antipsychotic (AP) medication and co-morbid substance use disorder. ${ }^{5-7}$ The symptom exacerbation after onset has been frequently described as "relapse", but the precise word definition is under continuous debate. ${ }^{8}$ Nevertheless, recent reports have consistently indicated that the occurrence of symptom exacerbations in early-stage psychosis, regardless of reasons, could result in brain changes, ${ }^{9,10}$ which likely underlies the poorer disease outcome. A pioneer study described that relapse duration was related to significant decreases in both total cerebral volume changes and frontal brain measures. ${ }^{9}$ This study implies that extended periods of relapse may have a negative effect on brain integrity in schizophrenia, suggesting the importance of implementing proactive measures that may prevent symptom exacerbation and improve treatment adherence. This observation is consistent with a paper that reported differences in functional connectivity among different stages of psychosis, based on the staging model proposed. ${ }^{10}$ Furthermore, a more recent paper reported adverse effects of symptomatic exacerbation, independent of the effects of AP medications, on brain structure. ${ }^{11}$

Although these seminal studies have tried to link brain changes with symptomatic exacerbation in early-stage psychosis, the present study aimed to address this relationship in a way that can be expanded in scalable and multi-institutional projects. To address this goal, we applied a crosssectional study design with the use of medical records in a retrospective manner. We used hospitalization due to deterioration of psychosis, as a simple and objective outcome indicator of 
symptom exacerbation in early-stage psychosis after onset. Accordingly, we used our cohort of early-stage psychosis ${ }^{12-16}$ and investigated their data of whole brain resting-state functional magnetic resonance imaging (rs-fMRI).

\section{METHODS}

\section{Study cohort}

We recruited patients within 24 months after onset, and confirmed diagnoses of psychotic disorders using the Structural Clinical Interview for DSM-IV Patient Edition (SCID). ${ }^{17}$ Exclusion criteria included a history of head trauma, nasal trauma, nasal surgery, neurologic disorder, cancer, viral infection, and reported history of intellectual disability. Additionally, participants with an estimated IQ below 70 on the Hopkins Adult Reading Test were excluded. ${ }^{18}$ Patients who reported active substance abuse or produced a urine drug screen positive for illicit substance use, except cannabis, were excluded. Individuals who were pregnant or taking anti-inflammatory agents or experienced any other psychiatric conditions were also excluded. Healthy controls (HCs) who had psychiatric diagnosis or a family history of schizophrenia-spectrum disorder were excluded. Positive and negative symptoms (presence, severity) of patients were evaluated with the Scale for the Assessment of Negative Symptoms (SANS) and the Assessment of Positive Symptoms (SAPS). ${ }^{19}$ See our past publications using the same cohort (the name might be first episode psychosis cohort) for more detail. ${ }^{12-16}$ This study was approved by the Johns Hopkins Medicine Institutional Review Boards and in accordance with The Code of Ethics of the World Medical Association (1964 Declaration of Helsinki). All study participants provided written informed consent. Parental consent and assent were obtained for all participants under the age of 18 years. 


\section{Clinical characterization}

Multiple board-certified psychiatrists assessed self-reports and the Johns Hopkins electronic medical database for the patients. We categorized patients in this cohort into two groups based on psychiatric hospitalization due to psychotic exacerbation. The first group consisted of patients who experienced no psychiatric hospitalization(s) between onset and study enrollment (P1), whereas the second group consisted of patients who experienced psychiatric hospitalization(s) due to psychotic exacerbration between onset and study enrollment (P2). We assessed selfreports and the Johns Hopkins electronic medical database for the patients to make sure that those hospitalizations are directly associated with symptom exacerbation. In addition, we carefully examined medical records for treatment adherence, duration between hospitalizations, and cannabis use.

We excluded 4 patients in P2, because of unclear medical history and hospitalization records. In addition, 1 patient in P2 with outlying brain imaging data (see "3-Tesla MRI brain imaging" section) was also excluded from the study. Therefore, 94 healthy controls (HC) and 85 patients (54 in P1 and 31 in P2) were analyzed in the present study. These patients include those with schizophrenia $(n=43)$, schizoaffective disorder $(n=14)$, schizophreniform disorder $(n=2)$, bipolar disorder with psychosis $(\mathrm{n}=19)$, major depressive disorder with psychotic features $(\mathrm{n}=$ 5), and not otherwise specified psychotic disorder $(n=2)$. In some analyses, the patients were also divided as non-affective group $(n=57)$ and affective group $(n=23)$ : the former includes schizophrenia and schizoaffective disorder, whereas the latter includes bipolar disorder with psychotic features and major depression disorder with psychotic features. We did not include 
schizophreniform disorder and not otherwise specified psychotic disorder in this dichotomic categorization.

The AP dosages were converted to chlorpromazine equivalents (CPZ) using the defined daily dose method..$^{20}$ Duration of illness (DOI) (between onset and rs-fMRI scan), duration of untreated psychosis (DUP) (between onset and first AP usage), and CPZ doses were obtained through self-reports and confirmed through the Johns Hopkins electronic medical database. In the overall patient group, 5 were treated with first-generation APs, 67 with second generation APs, and 6 were taking a combination of both first and second-generation APs. Seven patients were unmedicated at the first visit.

\section{3-Tesla MRI brain imaging}

Study participants were scanned using a Phillips 3T MRI scanner. Details about data acquisition and processing have been reported in our previous publications. ${ }^{16,21}$ Speficically, the rs-fMRI data were obtained with the following parameters: axial orientation, original matrix 80x80, 36 slices, voxel size 3x3x4 mm, TR/TE 2000/30 ms, 210 timepoints.

Rs-fMRI time courses were realigned to the mean image using rigid body registration, by following standard procedures to minimize the effects of head motion,. Motion and intensity outlier frames were identified and excluded using the Artifact Detection Tools (ART) toolbox. To further limit the potential confounding effects of head motion on this study, framewise displacement (FD) was calculated using six motion parameters, ${ }^{22}$ and one study participant with 
a FD larger than 0.3 was excluded from analysis. ${ }^{23,24}$ Furthermore, FD smaller than 0.3 was adjusted in downstream analysis (see details in "Statistical analysis" section).

MRIcloud (www.MRICloud.org) was used to segment and process structural MRI images. ${ }^{1}$ The detailed protocols for obtaining and processing MRI scans are described in our previous publication. ${ }^{2}$ High resolution T1 weighted image (magnetization-prepared rapid acquisition with gradient echo, MPRAGE) from each participant was parceled using MRICloud resulting in the definition of 78 cortical regions of interest (ROIs) (Table S1). For the segmentation, we used a multi-atlas set that matched with the demography of study participants in our cohort. The MPRAGE and the respective segmentation was then warped to the rs-fMRI. Through this process, we obtained 3003 resting state correlations (between the 78 cortical ROIs), that were then Fisher z-transformed. This approach reduces the image dimensionality from the voxel level from the order of billions to the order of hundreds, ${ }^{25}$ which results in an increase the statistical power and improve interpretation of analysis results. Furthermore, we performed graph theory analysis using the $\mathrm{R}$ brainGraph package and obtained global efficiency and nodal efficiency for downstream analyses.

\section{Statistical analysis}

R 3.5.1 and SPSS v24.0 were used to perform statistical analysis. Group comparisons of demographic and clinical data were calculated using independent t-tests for continuous variables, and chi-squared tests for categorical variables. 
Partial Pearson correlation analysis between clinical variables and brain imaging measures was conducted to evaluate potential confounding effects of clinical variables to brain imaging measures. Clinical variables include CPZ dose, DUP, DOI, and SAPS/SANS total scores, whereas brain imaging measures include the data from rs-FC, global efficiency, and nodal efficiency. The correlation analysis was conducted, controlling for age, gender, race, handedness, smoking status, and FD. The Benjamin-Hochberg $(\mathrm{BH})$ procedure, a method for controlling the false discovery rate (FDR), was performed for multiple comparison correction, ${ }^{26}$ and adjusted pvalues were presented as q-values. Brain imaging measures with sigifnicant correlation with clinical variables after multiple comparision correction were excluded from downstream analysis.

General linear regression, a statistical method often used to compare group differences or correlations between variables while controlling confounding factors, ${ }^{27,28}$ was performed to study differences in brain imaging measures between groups. Age, gender, race, handedness, smoking status, and FD were included as covariates in linear regression. Brain imaging measures with q-values smaller than 0.05 were considered as significant. Cross-group progression in significant brain imaging measures identified by general linear regression was tested with the Jonckheere-Terpstra test for ordered alternative hypotheses. ${ }^{10,29}$

\section{RESULTS}

\section{Demographic and clinical data}

First, we compared demographic characteristics among HC, P1, and P2 groups. Age and

handedness were not significantly different, whereas gender, race, and smoking status were 
different (Table 1). All these demographic variables were considered in the data analysis of rsfMRI measures. Next, we compared demographic characteristics between P1 group and P2 group. All these demographic characteristics were not different between P1 group and P2 group

(Table 1). Among key clinical characteristics, shorter DOI and lower AP doses were observed in P1 group, compared with P2 group (Table 2). P2 group had, although not significant, a higher mean value of the SAPS total score compared with P1 group $(\mathrm{p}=0.06)$. When subdividing patients into affective and non-affective cases, we didn't observe any significant difference in the proportion of $\mathrm{P} 1$ and $\mathrm{P} 2$ bewteen affective and non-affective cases (14 affective and 36 nonaffective cases for P1 and 9 affective and 21 non-affective cases for P2) (Table 2).

\section{Correlations between brain imaging measures and clinical variables}

We hypothesized that there are alterations in brain connectivity associated with symptom exacerbation. To test our hypothesis, we analyzed three complementary brain imaging measures derived from rs-fMRI scans: (i) global efficiency, which measures the overall capacity for parallel information transfer and integration at the systems level; ${ }^{30}$ (ii) nodal efficiency, which assesses the effectiveness of information exchange among the immediate neighbors of a given brain region; ${ }^{31}$ and (iii) rs-FC, which is the direct rs-fMRI correlation between pairs of regions and evaluates the ability of information transfer between two ROIs.

We hypothesized that clinical variables (CPZ dose, DUP, DOI, and SAPS/SANS total scores) may potentially affect some brain imaging measures. These measures that are significantly correlated with the clinical variables may not be relevantly used for the analyses that look for the association bewteen symptom exacerbation and brain connectivity. To avoid this problem, we 
first investigated correlations between the clinical variables and brain imaging measures. After multiple comparision correction, we underscored that the measures from the right caudate nucleus $(\mathrm{p}=1.90 \mathrm{E}-4, \mathrm{q}=8.26 \mathrm{E}-3)$ and left inferior occipital gyrus $(\mathrm{p}=2.10 \mathrm{E}-4, \mathrm{q}=8.27 \mathrm{E}-3)$ were significantly correlated with $\mathrm{CPZ}$ dose in nodal efficiency. No other significant correlations were observed bewteen the clinical variables and brain imaging measures of global efficiency, nodal efficiency, and rs-FC. Accordingly, we excluded the right caudate nucleus and left inferior occipital gyrus in nodal efficiency from downstream analysis.

\section{Group comparison between overall patient group and HC group}

We observed a significant decrease in global efficiency in the overall patient group compared to $\mathrm{HC}$ group (mean patient $=3.64$, mean $\mathrm{HC}=3.93, \mathrm{p}=0.03$ ). There were no brain regions with significant differences in nodal efficiency between the overall patient group and HC group (Figure 1). We observed a significant difference in 60 rs-FCs between the overall patient group and HC group after multiple comparison correction (Figure 1, Table S2).

\section{Group comparisons between HC, P1, and P2 groups}

We did not observe a significant difference in the mean of global efficiency between P1 and HC groups $(\mathrm{P} 1=3.65, \mathrm{HC}=3.93, \mathrm{p}=0.12)$, but observed a significant decrease of global efficiency in $\mathrm{P} 2$ group compared to $\mathrm{HC}$ group $(\mathrm{P} 2=3.62, \mathrm{HC}=3.93, \mathrm{p}=0.04)$. Although we did not observe significant differences in global efficiencies between P1 and P2 groups, JonckheereTerpstra test showed that there was a significant decrease $(p=0.04)$ in the mean values of global efficiency from $\mathrm{HC}$ to $\mathrm{P} 2$ group $(\mathrm{HC}>\mathrm{P} 1>\mathrm{P} 2)$. 
Consistently, for nodal efficiency, there were 17 brain regions with significantly decreased nodal efficiency in P2 group compared to HC group after multiple comparison correction (Figure 1). These brain regions included the bilateral dorsal anterior cingulate cortex (dorsal_ACC), left fusiform gyrus (FuG), left inferior frontal gyrus (IFG) opercularis, bilateral lingual gyrus (LG), left middle frontal gyrus (MFG), left middle occipital gyrus (MOG), bilateral middle temporal gyrus (MTG), right superior frontal gyrus_prefrontal cortex (SFG_PFC), bilateral superior occipital gyrus (SOG), left superior parietal gyrus (SPG), left superior temporal gyrus pole (STG_pole), left subcallosal ACC, and right thalamus (Table S3). We didn't observe significant differences in nodal efficiencies between P1 and HC groups, as well as those between P1 and P2 groups. Finally, Jonckheere-Terpstra test showed that there were significant decreasing trends in nodal efficiency from the $\mathrm{HC}$ to $\mathrm{P} 2$ group $(\mathrm{HC}>\mathrm{P} 1>\mathrm{P} 2)$ in these brain regions except in the left STG_pole (Table S4).

Next, we made group comparisons for rs-FC between P2 group, P1 group, and HC group. Among 3003 rs-FCs between 78 regions segmented by the MRIcloud, we observed that 131 rsFCs between 45 regions were significantly altered in P2 group when compared to HC group after multiple comparison correction (Figure 1, Table S5). The bilateral dorsal ACC (dACC), left supramarginal gyrus (SMG), left SOG, right inferior occipital gyrus (IOG), bilateral precentral gyrus (PrCG), right cerebellum, and bilateral thalamus were top 10 regions in regard to the number of significant rs-FCs between P2 and HC groups (Figure 2). In contrast, no rs-FCs were significantly different after multiple comparison correction between P1 and HC groups, as well as between $\mathrm{P} 1$ and $\mathrm{P} 2$ groups. 


\section{Brain regions associated with symptom exacerbation after onset in early-stage psychosis}

In the effort of integrating the results of group comparison between overall patient group and HC group with those of group comparisons between HC, P1, and P2 groups, we found that $84 \%$ (51 out of 60) rs-FCs that were different between the overall patient group and HC group were also underscored in the comparison between P2 group and $\mathrm{HC}$ groups, suggesting that P2 group who experienced symptom exacerbation(s) after onset may be the driver of the alterations observed in overall patient group compared to HC group.

Accordingly, we looked for overlapped brain regions that were commonly underscored both in nodal efficiency and rs-FC when compared between P2 group and HC group (Figure 1). As a result, we found bilateral $\mathrm{dACC}$ and right thalamus were highlighted. These brain regions are likely to be associated with symptom exacerbation. Note that the nodal efficiency in these these brain regions was reduced in $\mathrm{P} 2$ compared to $\mathrm{HC}$ group (Table S3) and showed a decreasing trend from HC to P2 (HC>P1>P2) (Figure 3A, Table S4). The rs-FCs involving the dACC and right thalamus that we highlighted here participated in several major large-scale brain networks (Figure 3B): for example, the right thalamus in the subcortical network, and the dACC in the salience network. In addition, the right thalamus showed increased correlation with the left MTG in the default mode network, the right SPG in the somatosensory network, and the SOG, MOG, IOG, FuG, LG, and cuneus $(\mathrm{Cu})$ in the visual network. The dACC also showed increased correlations with brain regions in the visual network (SOG, IOG, FuG, LG, and $\mathrm{Cu}$ ) as well as those with the right cerebellum. Together, we specifically underscored brain alterations in the right thalamus and bilateral dACC in the context of several major large-scale brain networks, that are associated with symptom exacerbation. 


\section{Discussion}

In the present study, we observed significant differences in gray matter connectivity between patients who experienced symptom exacerbation in early-stage psychosis and HCs. We evaluated three complementary metrics derived from the rs-fMRI data (global efficiency, nodal efficiency, and rs-FC) and obtained consistent results across all three regardless of the effects of DOI, DUP, AP medication or symptomatic features. Moreover, two key regions, the thalamus and dACC, were significantly altered in patients who experiences symptom exacerbation. These findings imply progressive brain changed associated with the disease, in particular symptom exacerbation.

Staging model of psychosis indicated that white matter connectivity is similar to controls in patients who only experienced first episode (stage II), whereas differences were observed in relapsing and incomplete remission patients (stage III). ${ }^{10}$ Their study also reported a decreasing trend of the global brain efficiency (HC>stage II >stage III) and decrease in nodal efficiency in the left SPG (white matter) in the stage III. Meanwhile, the present study showed a decreasing trend in the global brain efficiency $(\mathrm{HC}>\mathrm{P} 1>\mathrm{P} 2)$ and a significant decrease in nodal efficiency in the same brain region (left SPG) in P2 patients.

The brain regions with a decrease in nodal efficiency associated with symptom exacerbation shown in the present study participate in several large-scale brain networks, such as salience, subcortical, and default mode networks. Several studies have reported a reduction of the nodal efficiency in these networks across psychotic disorders, particularly in association with cognitive 
impairment and psychotic manifestation. ${ }^{32,33}$ These reports could be consistent with our observation in the nodal efficiency reduction associated with symptom exacerbation in earlystage psychosis, which provides patients with poorer functionality.

We pinned down that the thalamus and AACC could be important brain regions associated with symptom exacerbation in early-stage psychosis. These brain regions have been suggested as core pathological hubs involved in sensory, emotional, and cognitive processes, ${ }^{34,35}$ and have been examined in psychosis research. ${ }^{36-39}$ In particular, abnormalities in molecular brain imaging have also frequently underscored the dACC and thalamus in patients with psychosis. ${ }^{14,40-42}$ Deficits in a major antioxidant glutathione in the dACC have been specifically associated with treatment refractory patients with psychosis. ${ }^{43,44} \mathrm{~A}$ large meta-analysis that compared brain alterations in first episode psychosis and chronic patients highlighted an increase in thalamic connectivity only in later stage of illness but not in first episode psychosis, suggesting thalamic alterations may be associated with disease progression. ${ }^{45}$

Relapse is a complex condition to which many factors, such as AP medication cessation, substance abuse, stressful life events, may contribute. ${ }^{46}$ It is difficult to precisely address relapse in a cross-sectional study, whereas a longitudinal study design is expected. A systematic review regarding relapse in schizophrenia reported that hospitalization was the most widely used factor as a proxy for relapse by representing symptom exacerbation ( $62 \%$ of publications). ${ }^{47}$ The merit of using hospitalization is that it is objective, scalable, quantitative, and good as a proxy for large multi-institutional studies. ${ }^{48}$ Taken all together, in this cross-sectional study that is expected to be a prototype for scalable and multi-institutional projects (including data from multiple countries) 
in near future, we focused on symptom exacerbation after onset by using hospitalization as the key outcome measure for this. We acknowledge potential caveats in interpreting hospitalization as an endpoint for symptom exacerbation, because other factors such suicidality (versus psychotic symptoms) and family pressure may play a role. At least in the present study, we aimed to minimize this concern by using electronic medical database effectively.

\section{Acknowledgements:}

This study is supported by NIH grants MH-092443, MH-094268, MH-105660, and MH-107730;

foundation grants from Stanley and RUSK/S-R (to AS), an award from Brain and Behavior Research Foundation (to KY), and a Fulbright fellowship (to MM). Study recruitment was in part

funded by Mitsubishi Tanabe Pharma Corporation. The authors also appreciate Ms. Yukiko Lema and Dr. Mellisa A Landek-Salgado.

\section{Conflict of interest:}

All authors have nothing to disclose. 


\section{References}

1. Owen MJ, Sawa A, Mortensen PB. Schizophrenia. Lancet 2016; 388:86-97.

2. Kahn RS, Sommer IE, Murray RM, Meyer-Lindenberg A, Weinberger DR, Cannon TD, et al. Schizophrenia. Nat Rev Dis Primers 2015; 1:15067.

3. Suvisaari J, Mantere O, Keinanen J, Mantyla T, Rikandi E, Lindgren M, et al. Is It Possible to Predict the Future in First-Episode Psychosis? Front Psychiatry 2018; 9:580.

4. Wunderink L, van Bebber J, Sytema S, Boonstra N, Meijer RR, Wigman JTW. Negative symptoms predict high relapse rates and both predict less favorable functional outcome in first episode psychosis, independent of treatment strategy. Schizophr Res 2020; 216:192199.

5. Alvarez-Jimenez M, Priede A, Hetrick SE, Bendall S, Killackey E, Parker AG, et al. Risk factors for relapse following treatment for first episode psychosis: a systematic review and meta-analysis of longitudinal studies. Schizophr Res 2012; 139:116-128.

6. Caseiro O, Perez-Iglesias R, Mata I, Martinez-Garcia O, Pelayo-Teran JM, TabaresSeisdedos R, et al. Predicting relapse after a first episode of non-affective psychosis: a three-year follow-up study. J Psychiatr Res 2012; 46:1099-1105.

7. Bowtell M, Ratheesh A, McGorry P, Killackey E, O'Donoghue B. Clinical and demographic predictors of continuing remission or relapse following discontinuation of antipsychotic medication after a first episode of psychosis. A systematic review. Schizophr Res 2018; 197:9-18.

8. Csernansky JG, Mahmoud R, Brenner R, Risperidone USASG. A comparison of risperidone and haloperidol for the prevention of relapse in patients with schizophrenia. $N$ Engl J Med 2002; 346:16-22. 
9. Andreasen NC, Liu D, Ziebell S, Vora A, Ho BC. Relapse duration, treatment intensity, and brain tissue loss in schizophrenia: a prospective longitudinal MRI study. Am J Psychiatry 2013; 170:609-615.

10. Griffa A, Baumann PS, Klauser P, Mullier E, Cleusix M, Jenni R, et al. Brain connectivity alterations in early psychosis: from clinical to neuroimaging staging. Transl Psychiatry 2019; 9:62.

11. Voineskos AN, Mulsant BH, Dickie EW, Neufeld NH, Rothschild AJ, Whyte EM, et al. Effects of Antipsychotic Medication on Brain Structure in Patients With Major Depressive Disorder and Psychotic Features: Neuroimaging Findings in the Context of a Randomized Placebo-Controlled Clinical Trial. JAMA Psychiatry 2020; 77:674-683.

12. Kamath V, Lasutschinkow $P$, Ishizuka K, Sawa A. Olfactory Functioning in FirstEpisode Psychosis. Schizophr Bull 2018; 44:672-680.

13. Coughlin J, Du Y, Crawford JL, Rubin LH, Behnam Azad B, Lesniak WG, et al. The availability of the alpha7 nicotinic acetylcholine receptor in recent-onset psychosis: a study using (18)F-ASEM PET. J Nucl Med 2018.

14. Wang AM, Pradhan S, Coughlin JM, Trivedi A, DuBois SL, Crawford JL, et al. Assessing Brain Metabolism With 7-T Proton Magnetic Resonance Spectroscopy in Patients With First-Episode Psychosis. JAMA Psychiatry 2019; 76:314-323.

15. Narita Z, Yang K, Kuga H, Piancharoen P, Etyemez S, Faria AV, et al. Face processing of social cognition in patients with first episode psychosis: Its deficits and association with subcallosal anterior cingulate cortex. Schizophr Res 2021 [in press]. 
16. Faria AV, Zhao Y, Ye C, Hsu J, Yang K, Cifuentes E, et al. Multimodal MRI assessment for first episode psychosis: A major change in the thalamus and an efficient stratification of a subgroup. Hum Brain Mapp 2021; 42:1034-1053.

17. First MB, Spitzer RL, Gibbon M, Williams JBW. Structured Clinical Interview for DSMIV - Patient Edition (SCID-P, Version 2.0). : New York, NY: New York State Psychiatric Institute; 1996.

18. Schretlen DJ, Winicki JM, Meyer SM, Testa SM, Pearlson GD, Gordon B. Development, psychometric properties, and validity of the hopkins adult reading test (HART). Clin Neuropsychol 2009; 23:926-943.

19. Andreasen NC. Methods for assessing positive and negative symptoms. Mod Probl Pharmacopsychiatry 1990; 24:73-88.

20. Leucht S, Samara M, Heres S, Davis JM. Dose Equivalents for Antipsychotic Drugs: The DDD Method. Schizophr Bull 2016; 42 (Suppl 1):S90-94.

21. Mori S, Wu D, Ceritoglu C, Li Y, Kolasny A, Vaillant MA, et al. MRICloud: Delivering High-Throughput MRI Neuroinformatics as Cloud-Based Software as a Service. Comput Sci Eng 2016; 18:21-35.

22. Power JD, Barnes KA, Snyder AZ, Schlaggar BL, Petersen SE. Spurious but systematic correlations in functional connectivity MRI networks arise from subject motion. Neuroimage 2012; 59:2142-2154.

23. Achterberg M, van der Meulen M. Genetic and environmental influences on MRI scan quantity and quality. Dev Cogn Neurosci 2019; 38:100667. 
24. DeSerisy M, Musial A, Comer JS, Roy AK. Functional connectivity of the anterior insula associated with intolerance of uncertainty in youth. Cogn Affect Behav Neurosci 2020; 20:493-502.

25. Faria AV, Liang Z, Miller MI, Mori S. Brain MRI Pattern Recognition Translated to Clinical Scenarios. Front Neurosci 2017; 11:578.

26. Glickman ME, Rao SR, Schultz MR. False discovery rate control is a recommended alternative to Bonferroni-type adjustments in health studies. J Clin Epidemiol 2014; $67: 850-857$

27. Morgan SL, Morgan SL, SpringerLink. Handbook of Causal Analysis for Social Research. 1st 2013. ed. Dordrecht: Springer Netherlands : Imprint: Springer; 2013.

28. McNamee R. Regression modelling and other methods to control confounding. Occup Environ Med 2005; 62:500-506, 472.

29. Bewick V, Cheek L, Ball J. Statistics review 10: further nonparametric methods. Crit Care 2004; 8:196-199.

30. Bullmore E, Sporns O. The economy of brain network organization. Nat Rev Neurosci $2012 ; 13: 336-349$.

31. Latora V, Marchiori M. Efficient behavior of small-world networks. Phys Rev Lett 2001; $87: 198701$.

32. Sheffield JM, Kandala S, Tamminga CA, Pearlson GD, Keshavan MS, Sweeney JA, et al. Transdiagnostic Associations Between Functional Brain Network Integrity and Cognition. JAMA Psychiatry 2017; 74:605-613.

33. Zhang W, Lei D, Keedy SK, Ivleva EI, Eum S, Yao L, et al. Brain gray matter network organization in psychotic disorders. Neuropsychopharmacology 2020; 45:666-674. 
34. Woodward ND, Karbasforoushan H, Heckers S. Thalamocortical dysconnectivity in schizophrenia. Am J Psychiatry 2012; 169:1092-1099.

35. Fornito A, Yucel M, Dean B, Wood SJ, Pantelis C. Anatomical abnormalities of the anterior cingulate cortex in schizophrenia: bridging the gap between neuroimaging and neuropathology. Schizophr Bull 2009; 35:973-993.

36. Woodward ND, Heckers S. Mapping Thalamocortical Functional Connectivity in Chronic and Early Stages of Psychotic Disorders. Biol Psychiatry 2016; 79:1016-1025.

37. Palaniyappan L, Simmonite M, White TP, Liddle EB, Liddle PF. Neural primacy of the salience processing system in schizophrenia. Neuron 2013; 79:814-828.

38. Anticevic A. Understanding the role of thalamic circuits in schizophrenia neuropathology. Schizophr Res 2017; 180:1-3.

39. Miyata J. Toward integrated understanding of salience in psychosis. Neurobiol Dis 2019; 131:104414.

40. Wang M, Barker PB, Cascella N, Coughlin JM, Nestadt G, Nucifora FC, et al. Longitudinal changes in brain metabolites in healthy subjects and patients with first episode psychosis (FEP): a 7-Tesla MRS study. bioRxiv 2020.

41. Bojesen KB, Broberg BV, Fagerlund B, Jessen K, Thomas MB, Sigvard A, et al. Associations Between Cognitive Function and Levels of Glutamatergic Metabolites and Gamma-Aminobutyric Acid in Antipsychotic-Naive Patients With Schizophrenia or Psychosis. Biol Psychiat 2021; 89:278-287.

42. Reid MA, Salibi N, White DM, Gawne TJ, Denney TS, Lahti AC. 7T Proton Magnetic Resonance Spectroscopy of the Anterior Cingulate Cortex in First-Episode Schizophrenia. Schizophr Bull 2019; 45:180-189. 
43. Dempster K, Jeon P, MacKinley M, Williamson P, Theberge J, Palaniyappan L. Early treatment response in first episode psychosis: a 7-T magnetic resonance spectroscopic study of glutathione and glutamate. Mol Psychiatr 2020; 25:1640-1650.

44. Yang K, Longo L, Narita Z, Cascella N, Nucifora FC, Jr., Coughlin J, et al. A multimodal study of a first episode psychosis cohort: potential markers of antipsychotic treatment resistance. Mol Psychiatr 2021 [in press].

45. Li T, Wang Q, Zhang J, Rolls ET, Yang W, Palaniyappan L, et al. Brain-Wide Analysis of Functional Connectivity in First-Episode and Chronic Stages of Schizophrenia. Schizophr Bull 2017; 43:436-448.

46. Taylor M, Jauhar S. Are we getting any better at staying better? The long view on relapse and recovery in first episode nonaffective psychosis and schizophrenia. Ther $A d v$ Psychopharmacol 2019; 9:2045125319870033.

47. Olivares JM, Sermon J, Hemels M, Schreiner A. Definitions and drivers of relapse in patients with schizophrenia: a systematic literature review. Ann Gen Psychiatry 2013; 12:32.

48. Addington DE, Patten SB, McKenzie E, Addington J. Relationship between relapse and hospitalization in first-episode psychosis. Psychiatr Serv 2013; 64:796-799. 


\section{Figure legends}

\section{Figure 1. Study design and key results}

We collected 3 brain imaging measures from rs-fMRI. For each measure, we conducted group comparisons to identify significant changes in the overall patient group, P1 group, and P2 group, compared with HC group, respectively. Numbers in the figure are the count of significant variables in group comparisons. By integrating all the analysis results, we pinned down 3 brain regions, the right thalamus and bilateral $\mathrm{dACC}$, which may be associated with symptom exacerbation after onset.

Figure 2. The number of significant rs-FC between $\mathrm{P2}$ and $\mathrm{HC}$ group of each brain region. We observed 131 rs-FCs of 45 regions that were significantly different between P2 and HC group. Top 10 brain regions with most number of significant rs-FCs are highglihted with black bars. Abbreviations for brain regions are listed in Table S1.

Figure 3. Brain regions associated with symptom exacerbation (bilateral dACC and left thalamus) after onset in early-stage psychosis.

A. Boxlot of bilateral dACC and right thalamus across P1, P2, and HC groups. The box represents standard deviation and the solid line in the middle of the box shows the mean value. Black dots represent individual subjects. Star means that Jonckheere-Terpstra test found a significant decreasing trend in nodal efficiency from the HC to $\mathrm{P} 2$ group $(\mathrm{HC}>\mathrm{P} 1>\mathrm{P} 2)$. B. Illustration of significant rs-FC between the bilateral dACC and right thalamus with other brain regions in the comparison between P2 and HC. Dots represent brain regions and lines represent connectivity between regions. 
bioRxiv preprint doi: https://doi.org/10.1101/2021.12.10.472143; this version posted December 14, 2021. The copyright holder for this preprint (which was not certified by peer review) is the author/funder. All rights reserved. No reuse allowed without permission.

Note: Please see the abbreviations for brain regions in Table S1. 
Table 1. Demographic data of study participants.

\begin{tabular}{|c|c|c|c|c|c|}
\hline & $\begin{array}{c}\mathbf{H C} \\
(n=94)\end{array}$ & $\begin{array}{c}\text { P1 } \\
(n=54)\end{array}$ & $\begin{array}{c}\text { P2 } \\
(n=31)\end{array}$ & $\begin{array}{c}\text { HC vs P1 vs P2 } \\
\text { p-value }\end{array}$ & $\begin{array}{l}\text { P1 vs P2 } \\
\text { p-value }\end{array}$ \\
\hline $\begin{array}{l}\text { Age } \\
(\text { mean } \pm \text { SD) }\end{array}$ & $23.5 \pm 3.8$ & $22.3 \pm 4.8$ & $22.4 \pm 4.2$ & 0.171 & 0.888 \\
\hline $\begin{array}{l}\text { Gender } \\
(\text { male \%) }\end{array}$ & 44.7 & 72.2 & 71.0 & 0.001 & 0.902 \\
\hline $\begin{array}{l}\text { Race } \\
\text { (white \%) }\end{array}$ & 37.2 & 57.4 & 35.5 & 0.038 & 0.052 \\
\hline $\begin{array}{l}\text { Smoking } \\
(\text { yes \%) }\end{array}$ & 4.3 & 24.1 & 32.3 & $2.400 \mathrm{E}-05$ & 0.454 \\
\hline $\begin{array}{l}\text { Handedness } \\
\text { (right \%) }\end{array}$ & 89.4 & 94.4 & 80.6 & 0.139 & 0.067 \\
\hline
\end{tabular}


Table 2. Clinical measures of early-stage psychosis patients.

\begin{tabular}{lccc}
\hline $\begin{array}{l}\text { Clinical } \\
\text { Measure }\end{array}$ & $\begin{array}{c}\text { P1 } \\
(\mathrm{n}=54)\end{array}$ & $\begin{array}{c}\mathbf{P 2} \\
(\mathrm{n}=31)\end{array}$ & $\begin{array}{c}\text { P1 vs P2 } \\
\text { p-value }\end{array}$ \\
\hline CPZ & $202.1 \pm 219.7$ & $312.9 \pm 265.6$ & $\mathbf{0 . 0 4 2}$ \\
DOI & $12.4 \pm 9.1$ & $18.6 \pm 7.6$ & $\mathbf{0 . 0 0 2}$ \\
DUP & $4.3 \pm 7.5$ & $4.0 \pm 5.7$ & 0.841 \\
SAPS & $3.1 \pm 3.5$ & & 0.055 \\
SANS & & $4.7 \pm 4.2$ & \\
Affective group & $3.6 \pm 4.6$ & & 0.616 \\
\end{tabular}

Note: Values present mean \pm SD or percentage (\%). P-value $<0.05$ are presented in bold.

CPZ: chlorpromazine doses equivalents; DOI: duration of illness; DUP: duration of untreated psychosis; SAPS/SANS: total scores of positive/negative symptoms scales. 


\section{Figure 1}

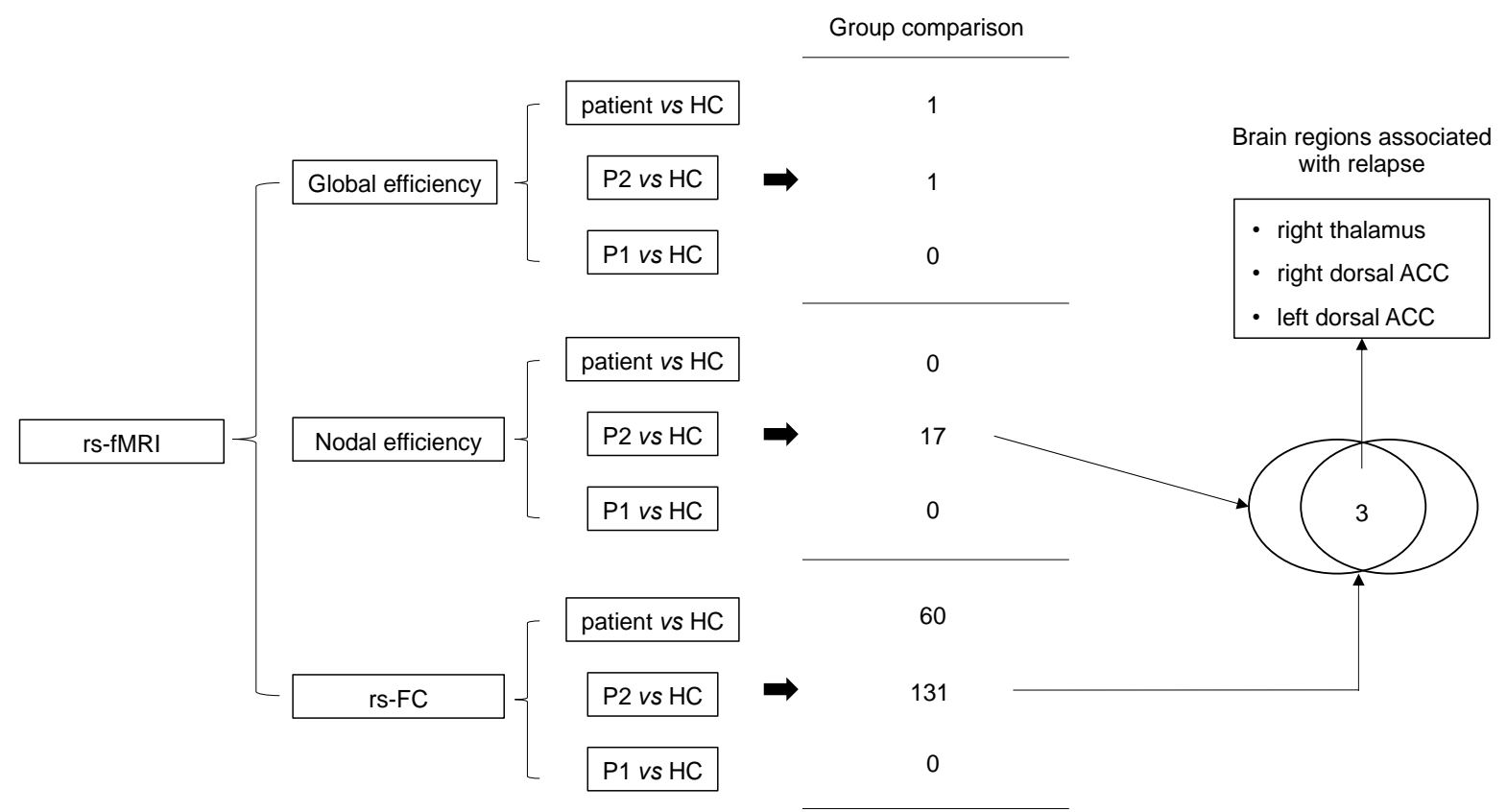


bioRxiv preprint doi: https://doi.org/10.1101/2021.12.10.472143; this version posted December 14, 2021. The copyright holder for this preprint (which was not certified by peer review) is the author/funder. All rights reserved. No reuse allowed without permission.

Figure 2

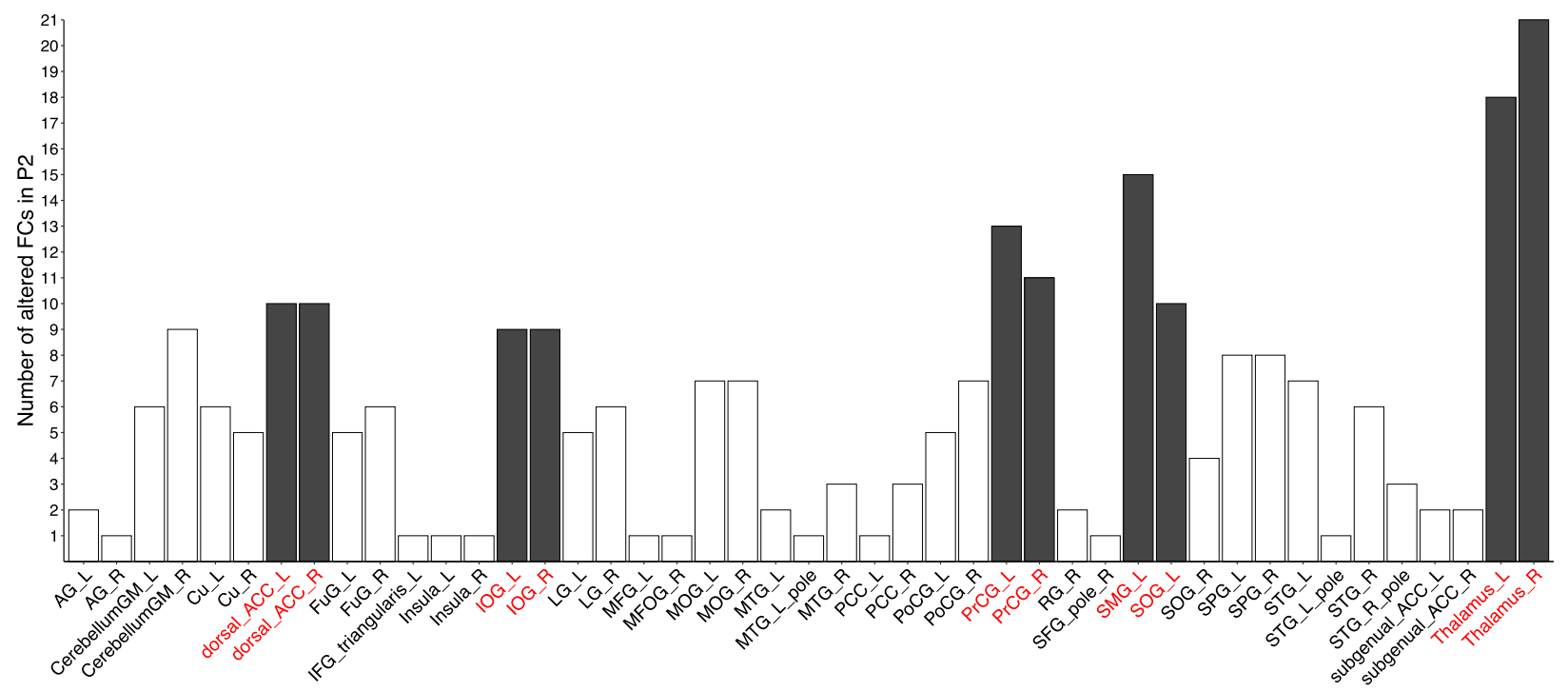




\section{Figure 3}
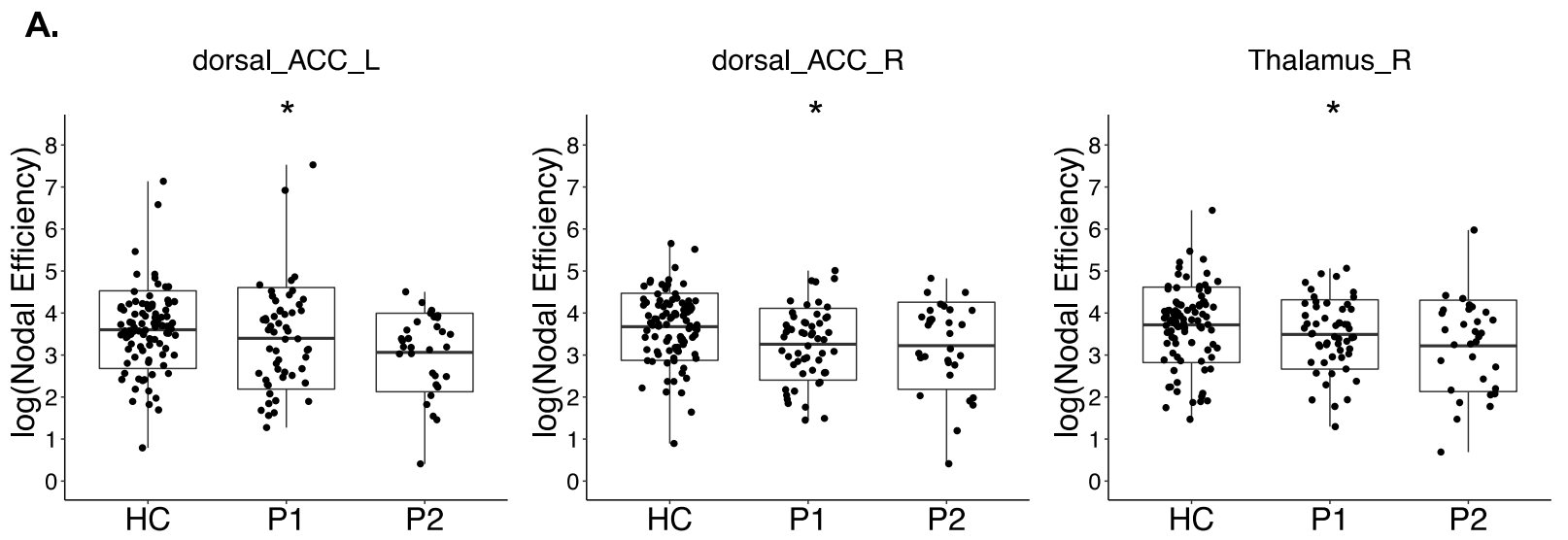

B.

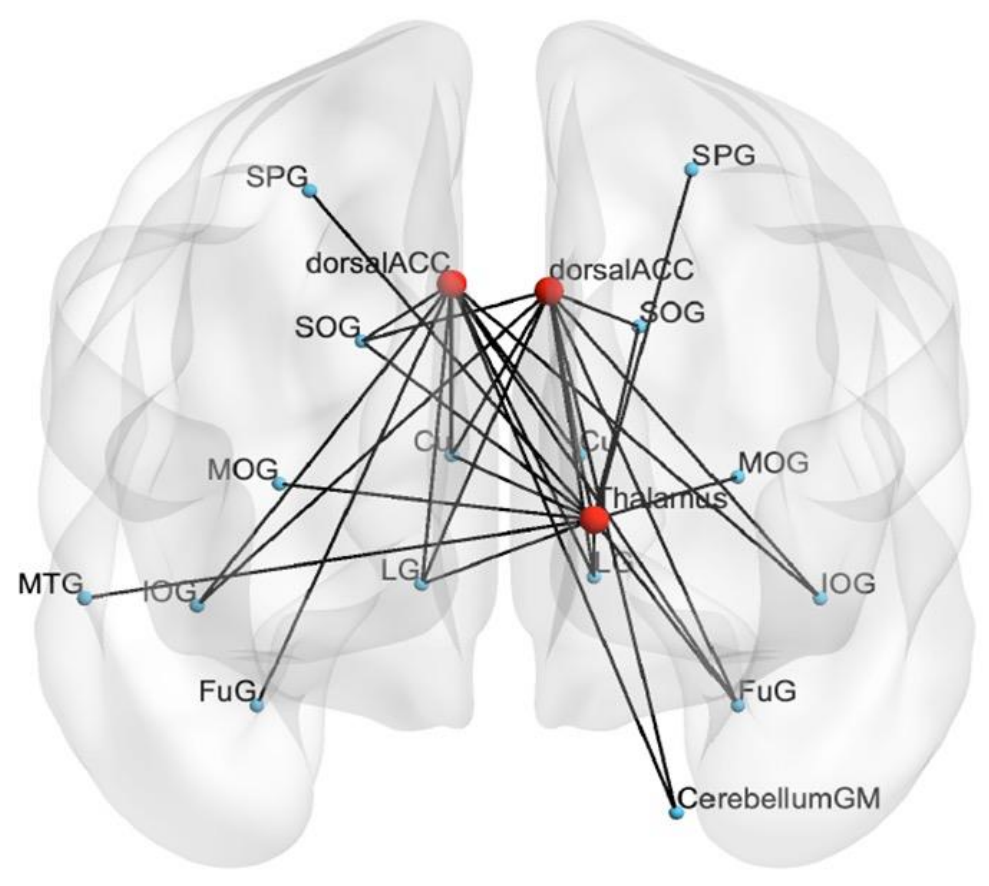

